\title{
Development of Polymer and Surfactant Based Naringenin Nanosuspension for Improvement of Stability, Antioxidant, and Antitumour Activity
}

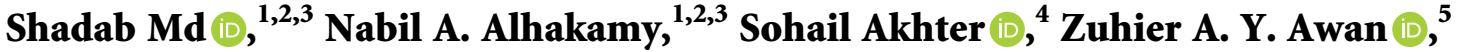 \\ Hibah M. Aldawsari, ${ }^{1,2}$ Waleed S. Alharbi, ${ }^{1}$ Anzarul Haque, ${ }^{6}$ Hira Choudhury, ${ }^{7}$ \\ and Ponnurengam Malliappan Sivakumar ${ }^{8}$ \\ ${ }^{1}$ Department of Pharmaceutics, Faculty of Pharmacy, King Abdulaziz University, Jeddah, Saudi Arabia \\ ${ }^{2}$ Center of Excellence for Drug Research \& Pharmaceutical Industries, King Abdulaziz University, Jeddah, Saudi Arabia \\ ${ }^{3}$ King Fahd Medical Research Center, King Abdulaziz University, Jeddah, Saudi Arabia \\ ${ }^{4}$ Department of New Product Development, Combination Product \& Devices, Global R\&D, Teva Pharmaceuticals, Runcorn, UK \\ ${ }^{5}$ Department of Medicine and Biochemistry, Faculty of Medicine, King Abdulaziz University, Jeddah, Saudi Arabia \\ ${ }^{6}$ Department of Pharmacognosy, College of Pharmacy, Prince Sattam Bin Abdulaziz University, Al-kharj, Saudi Arabia \\ ${ }^{7}$ Department of Pharmaceutical Technology, School of Pharmacy, International Medical University, Jalan Jalil Perkasa, \\ Bukit Jalil, Kuala Lumpur 57000, Malaysia \\ ${ }^{8}$ Center for Molecular Biology, Institute of Research and Development, Duy Tan University, 03 Quang Trung, Da Nang, Vietnam
}

Correspondence should be addressed to Shadab Md; shadabmd1982@gmail.com and Ponnurengam Malliappan Sivakumar; sivamedchem@gmail.com

Received 25 November 2019; Revised 18 January 2020; Accepted 1 June 2020; Published 15 July 2020

Guest Editor: Wenji Li

Copyright (C) 2020 Shadab Md et al. This is an open access article distributed under the Creative Commons Attribution License, which permits unrestricted use, distribution, and reproduction in any medium, provided the original work is properly cited.

\begin{abstract}
Nanosuspensions are widely reported to enhance the solubility of poorly soluble drugs. In addition to enhancement in solubility, improvement of stability and therapeutic efficacy would be an added advantage. In the present study, premilling and subsequent high-pressure homogenization were carried out to produce naringenin nanosuspension. Hydroxypropyl methylcellulose and sodium dodecyl sulfate were evaluated for their performance as stabilizers under various homogenization cycles. The prepared nanosuspensions were studied for average particle size and size distribution, zeta potential, solubility, drug release, antioxidant activity, and in vitro antitumor activity. It was observed that both hydroxypropyl methylcellulose-stabilized nanosuspension and sodium dodecyl sulfate-stabilized nanosuspension produced an enhancement in physical stability, antioxidant potential, and in vitro cytotoxicity compared with naringenin. Furthermore, hydroxypropyl methylcellulose-stabilized nanosuspension was found to be better than sodium dodecyl sulfate-stabilized nanosuspension in terms of particle size and size distribution, storage stability, and drug release. This study showed that nanosuspension formulations could be a potential strategy for improving dissolution and antitumor activity of naringenin.
\end{abstract}

\section{Introduction}

Breast cancer has been the major cause of cancer-related deaths in women. Breast cancer can be treated by chemotherapy and other methods [1]. However, chemotherapy often produces serious side effects [1]. The need for new drugs has initiated the assessment of fruits, vegetables, herbs, and spices for anticancer properties [2], leading to studies on dietary phytochemicals [3]; these, in particular flavonoids, have been reported to inhibit the growth of different types of cancer cells by inhibiting cell proliferation and activating apoptosis [4]. Naringenin (NAR, 4',5,7-trihydroxyflavanone), a naturally 
occurring flavonoid present in citrus fruits, possesses antioxidant, hepatoprotective, anti-inflammatory, and anticarcinogenic effects $[5,6]$. Despite its therapeutic potential, NAR has not been developed for clinical use due to its low water solubility, low membrane permeability, instability in physiological media, first pass metabolism, and low bioavailability [5]. Several approaches have been tried to resolve the solubility problem, using cosolvents, micronization, and complexation with cyclodextrins; however, these techniques have limitations in enhancing solubility of drugs [7, 8]. Nanotechnology is being used increasingly for its pharmaceutical applications [9]. Nanosuspensions are carrier-free, submicron colloidal dispersions which consist of pure drugs and stabilizers [10]. They are produced using top-down techniques which comprise high-pressure homogenization (HPH) and media milling techniques [10]. The advantages of nanosuspensions include passive drug targeting and improved solubility, stability, and bioavailability, as well as lower drug systemic toxicity $[7,10]$. Many studies using nanotechnology drug formulations have been conducted, and some show promising results. NAR-loaded nanoparticles were reported to improve NAR release, which increases their hepatoprotective effects and anticancer potential [5, 6]. There have been no studies on the anticancer or antioxidant effects of NAR nanosuspensions, although other flavonoids, such as apigenin, puerarin, and diosmin, in the nanosuspension were shown, respectively, to have increased antioxidant activity [8], anticancer actions with lower toxicity [10], band-enhanced permeation, and dissolution characteristics for better drug delivery [11]. Therefore, evaluation of NAR nanosuspensions for antioxidant and antitumor activity on the breast cancer cell line was warranted. The objective of the present investigation was to develop and evaluate polymer- and surfactantbased nanosuspensions using HPH techniques. Short-term physical stability was assessed by storing different stabilized nanosuspensions at three different temperatures to reveal the most effective stabilizer. Antitumor activity of NAR nanosuspensions was examined in a breast cancer cell line (MCF-7 cells), and its in vitro antioxidant potential was determined and compared with free drug solution.

\section{Materials and Methods}

2.1. Materials. Naringenin drug (Sigma-Aldrich, USA) in powder form was used without further purification. Hydroxypropyl methylcellulose (HPMC) and sodium dodecyl sulfate (SDS) (Merck, Germany) were used as stabilizers for the nanosuspension. Ethanol (Merck, Germany), 2,2-diphenyl-1-picrylhydrazyl (DPPH), and gallic acid (Sigma-Aldrich, USA) were used. The breast cancer cell line (MCF-7 cells) was purchased from the American Type Culture Collection (ATCC) (Manassas, VA, USA). Dulbecco's Modified Eagle Medium (DMEM), fetal bovine serum, penicillin, streptomycin, and trypsin were purchased from $\mathrm{Gibco}^{\mathrm{TM}}$ (Invitrogen, Carlsbad, CA).

2.2. Preparation of NAR Nanosuspensions. For nanosuspension preparation, naringenin powder $(2 \% \mathrm{w} / \mathrm{v})$ was dispersed into two different aqueous stabilizer solutions

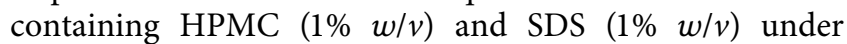
magnetic stirring. The mixture was disintegrated into microparticles using a high-shear homogenizer (Ultrturax T25, IKA, Germany) at 20,000 rpm for $5 \mathrm{~min}$. The microparticles were further disintegrated via high-pressure homogenization (IKA, Germany) for 6 homogenization cycles at 800 bar followed by 20 homogenization cycles at 1500 bar. Samples were collected after premilling, and different homogenization cycles at 1500 bar were considered for characterization study. The optimized nanosuspension was freeze-dried in the freeze dryer for $72 \mathrm{~h}$ using $5 \%(w / v)$ lactose as a cryoprotectant. The freeze-dried nanosuspensions were redispersed in distilled water before analysis.

\subsection{Characterization of Nanosuspensions}

2.3.1. Photon Correlation Spectroscopy (PCS). The particle size, polydispersity index (PDI), and zeta potential of the nanosuspension were measured by PCS using Zetasizer Nano ZSP (Malvern Instruments, UK). The prepared nanosuspensions were diluted with distilled water, and results were reported in triplicate.

\subsubsection{Transmission Electron Microscopy (TEM) and Scanning} Electron Microscopy (SEM). The actual particle size and morphology of optimized NAR nanosuspensions were investigated using TEM (JEOL-JEM1010, Japan) and SEM (Hitachi, Tokyo, Japan). A drop of the diluted nanosuspension was placed onto a carbon-coated copper grid, stained by adding a drop of $1 \%(w / w)$ phosphotungstic acid. After staining, samples were viewed using a TEM. Freezedried samples of NAR nanosuspensions were fixed onto aluminium stubs with a double-sided adhesive tape and were coated with gold for sample preparation before SEM observation.

2.4. Physical Stability of the Nanosuspension. For physical stability analysis, the nanosuspension was stored at three different temperatures $\left(4^{\circ} \mathrm{C}, 25^{\circ} \mathrm{C}\right.$, and $\left.40^{\circ} \mathrm{C}\right)$ for 30 days. The parameters were measured on days 7,14 , and 30 .

2.5. Solubility Studies. The saturation solubility studies of free NAR and two lyophilized nanosuspensions stabilized by HPMC and SDS stabilizers were tested in distilled water and phosphate buffer solution (PBS) ( $\mathrm{pH}$ 6.8) using a temperature-controlled water bath shaker. $10 \mathrm{mg}$ of the NAR sample, lyophilized NAR-NS-HPMC, and NAR-NS-SDS powder equivalent to $10 \mathrm{mg}$ of the pure NAR drug were added to $5 \mathrm{ml}$ distilled water and PBS ( $\mathrm{pH} 6.8)$. The temperature and speed were set at $37^{\circ} \mathrm{C}$ and $100 \mathrm{rpm}$, for $48 \mathrm{~h}$. Nanosuspensions were centrifuged at $4000 \mathrm{rpm}$ for $10 \mathrm{~min}$. The clear supernatant sample was obtained and passed through the $0.2 \mu \mathrm{m}$ membrane filter, and NAR content was analyzed by a UV spectrophotometer at $320 \mathrm{~nm}$. 
2.6. In Vitro Release Study. The in vitro study was carried out by transferring the prepared drug solution and nanosuspension into dialysis tubing, which was then immersed in phosphate buffer solution (PBS) $(100 \mathrm{~mL}, \mathrm{pH}$ 6.8) under magnetic stirring at $37^{\circ} \mathrm{C}$ at $100 \mathrm{rpm} .2 \mathrm{~mL}$ samples were withdrawn at different time intervals $(5,15,30,60$, and $120 \mathrm{~min}$ ) and replaced with the same volume of fresh PBS. The samples were filtered and analyzed using a UV spectrophotometer in triplicate. The percentage of drug released was plotted against time.

2.7. Determination of Antioxidant Activity. The antioxidant activity of NAR solution and nanosuspensions was measured using the method of Shaal and coworkers [8]. For plotting a standard curve, different concentrations $(10,25$, 50 , and $100 \mathrm{M}$ ) of DPPH in methanol were prepared. $0.2 \mathrm{~mL}$ of the NAR solution or nanosuspension was mixed with $2.8 \mathrm{~mL}$ of DPPH methanolic solution $(0.0355 \mathrm{~g} / 100 \mathrm{~mL})$ for each concentration, respectively. Gallic acid was used as a positive control. All the samples were kept for 30 minutes at $37^{\circ} \mathrm{C}$ followed by measuring the absorbance using a UV spectrophotometer at $520 \mathrm{~nm}$ in triplicate. The percentage inhibition of the DPPH free radical was calculated.

Inhibition of DPPH free radical $(\%)=($ absorbance control control - absorbance test/absorbance control $) \times 100$. Then, the (\%) inhibition of the DPPH free radical was plotted against the concentration of DPPH.

2.8. In Vitro Cytotoxicity Assessment. The in vitro cytotoxicity of free naringenin and naringenin nanosuspension was evaluated using the MTT assay. MCF-7 breast cancer cells were cultured in a humidified 5\% CO2/95\% atmosphere incubator at $37^{\circ} \mathrm{C}$. DMEM medium was used, containing $10 \%$ fetal bovine serum, $100 \mathrm{U} / \mathrm{mL}$ penicillin, and $100 \mathrm{ug} / \mathrm{mL}$ streptomycin. The medium was exchanged every 2-3 days. MCF-7 cells were seeded at a density of $5 \times 10^{3}$ cells per well in 96 -well plates at $37^{\circ} \mathrm{C}$ in a $5 \% \mathrm{CO}_{2}$ atmosphere for 24 hours in the same medium used for cell culture. The cells were then incubated with free naringenin or with naringenin nanosuspension at various concentrations for the indicated time periods. Controls were incubated in the same volume of culture media containing DMSO. $10 \mu \mathrm{l}$ of MTT was added to each well and allowed to incubate for an additional 4 hours. The maximum absorbance of each well was detected at $570 \mathrm{~nm}$ using a microplate reader.

2.9. Morphological Analysis of Cells by Phase-Contrast Microscopy. Morphological study by phase-contrast microscopy was undertaken according to a previous method [12]. Changes to the MCF-7 cells incubated in the 96-well plates at $100 \mu \mathrm{g} / \mathrm{mL}$ were observed using an inverted microscope (Nikon, Japan) after 24 and 48 hours of incubation.

2.10. Statistical Analysis. Results were presented as mean\pm standard deviation. Statistical analysis was conducted using the one-way ANOVA to evaluate the significance of differences between groups with $p<0.05$ as significant.

\section{Results and Discussion}

3.1. Preparation and Optimization of NAR Nanosuspensions. Premilling and subsequent high-pressure homogenization have been reported to produce stable drug nanosuspension [13]. A surfactant or polymer was necessary for stabilization of nanosuspensions during the high-pressure homogenization process. HPMC- and SDS-stabilized systems are reported for nanosuspensions [14-16]. In the present study, polymer-based (HPMC) and surfactant-based (SDS) stabilizers were selected for preparation of NAR nanosuspensions. HPMC and SDS are extensively used as excipients with no known toxicity [14-16]. NAR nanosuspension formulations were prepared using different stabilizer concentrations $(0.1 \%-1 \% \mathrm{w} / \mathrm{v})$. The effect of different concentrations of stabilizers on particle size, PDI, and zeta potential of NAR formulations is presented in Table 1. The results showed that, by decreasing the stabilizer concentration, there was a substantial increase in the particle size and PDI of the NAR nanosuspension. It could be due to poor dispersion of hydrophobic particles of NAR at low stabilizer concentration and formation of agglomerates [17]. $\mathrm{Ma}$ and coworkers also reported a similar observation [17]. In case of SDS, with increase in the concentration of stabilizers, the zeta potential value also increases significantly $(p<0.05)$ from $-25.86 \pm 1.72$ to $-40.63 \pm 2.23 \mathrm{mV}$ as shown in Table 1. However, different concentrations of HPMC did not change their zeta potential values significantly $(p<0.05)$ as compared with the SDS stabilizer [17]. The stabilizer concentration $1 \% \mathrm{w} / \mathrm{v}$ for HPMC- and SDS-stabilized nanosuspensions was optimized. The quality of the nanosuspension can depend on the pressure and number of cycles used in the method. The prepared nanosuspensions were further optimized based on pressure and the number of cycles.

3.2. Effect of Homogenization Cycle and Pressure on the NAR Nanosuspension. In the present study, we examined the effect of homogenization cycles $1,5,10,15$, and 20 in the preparation of nanosuspensions. In the case of nanosuspensions prepared with HPMC, the average particle size was found to decrease gradually from $615 \pm 10.2$ to $365 \pm 1.9 \mathrm{~nm}$ when the homogenization cycle was changed from 1 to 20 (Figure 1(a)). The average particle size of the HPMC-stabilized nanosuspension was well dependent on the homogenization cycle. A homogenization cycle of 10 produced a distinct effect on the particle size. It resulted in an average particle size of $489 \pm 5.5 \mathrm{~nm}$. Meanwhile, homogenization cycles of 1 and 5 produced more or less similar average particle size. Similarly, 15 and 20 produced particles with comparable size. It was noted that PDI also decreased when the homogenization cycle was changed from 1 to 20 . The PDI observed for 1,5 , and 10 cycles was comparable, while the PDI for 15 and 20 cycles was similar and much lower to the former ones.

The effect of homogenization cycles on the preparation of SDS-stabilized nanosuspensions is shown in Figure 1(b). In this case also, a gradual decrease in the average particle 
TABLE 1: Optimization parameters of NAR nanosuspensions stabilized by different concentrations of SDS and HPMC.

\begin{tabular}{|c|c|c|c|}
\hline Samples & Particle size $(\mathrm{nm})($ mean $\pm \mathrm{SD})$ & PDI $($ mean \pm SD $)$ & $\mathrm{Zp}(\mathrm{mV})($ mean $\pm \mathrm{SD})$ \\
\hline \multicolumn{4}{|c|}{ SDS conc. $(\% \mathrm{w} / \mathrm{v})$} \\
\hline F1 $(0.1)$ & $1251 \pm 14.9$ & $0.52 \pm 0.04$ & $-25.9 \pm 1.7$ \\
\hline F2 (0.5) & $674 \pm 6.9$ & $0.44 \pm 0.02$ & $-34.7 \pm 1.9$ \\
\hline F3 (1) & $460 \pm 7.3$ & $0.33 \pm 0.03$ & $-40.6 \pm 2.2$ \\
\hline \multicolumn{4}{|c|}{ HPMC conc. $(\% \mathrm{w} / \mathrm{v})$} \\
\hline $\mathrm{F} 4(0.1)$ & $1421 \pm 13.6$ & $0.58 \pm 0.05$ & $-5.8 \pm 0.4$ \\
\hline F5 (0.5) & $760 \pm 12.3$ & $0.45 \pm 0.03$ & $-7.1 \pm 0.8$ \\
\hline F6 (1) & $372 \pm 3.50$ & $0.32 \pm 0.04$ & $-8.8 \pm 0.5$ \\
\hline
\end{tabular}

Drug : stabilizer $(2: 1)$ ratio; 15 homogenization cycles and pressure 1500 bar were fixed.

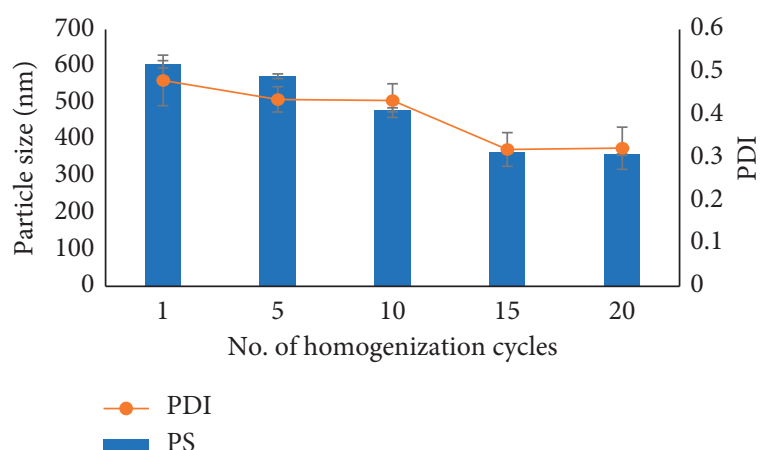

(a)

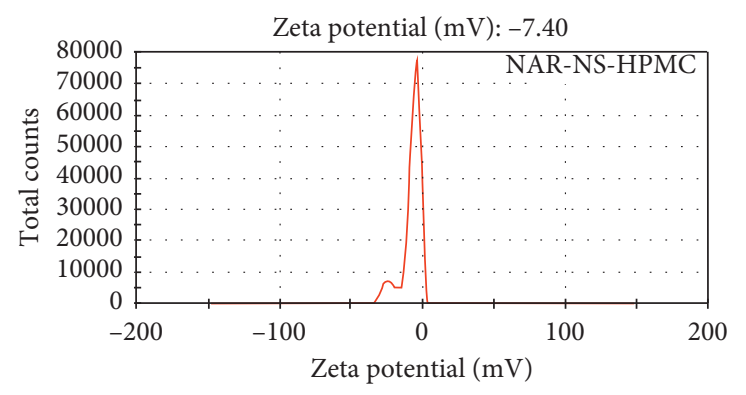

(c)

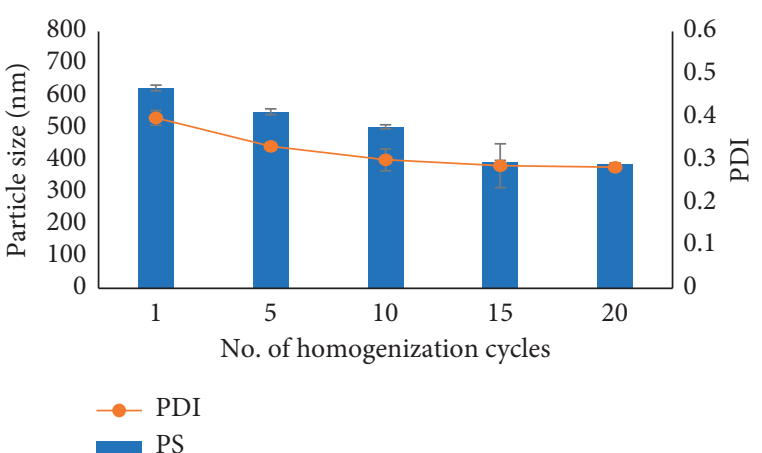

(b)

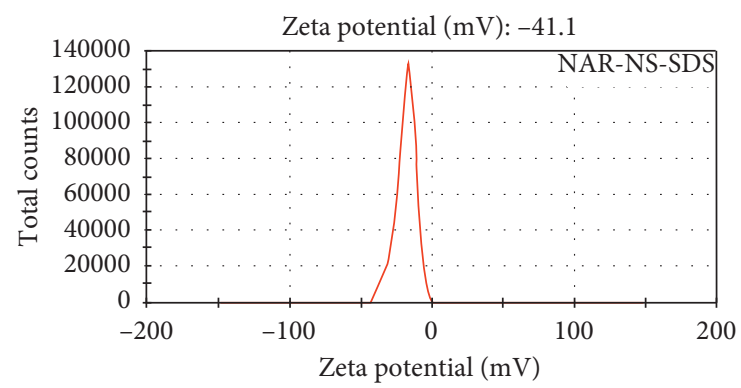

(d)

FIGURE 1: Mean particle size and polydispersity index (PDI) as a function of cycle numbers. 1, 5, 10, 15, and 20 homogenization cycles and pressure 1500 bar $(n=3)$ for the HPMC-stabilized NAR nanosuspension (a) and the SDS-stabilized NAR nanosuspension (b). (c, d) Zeta potential of HPMC- and SDS-stabilized NAR nanosuspensions.

size can be seen by increasing the homogenization cycle from 1 to 20 . SDS also produced similar particle sizes for 15 and 20 cycles; the average particle size was well below the values obtained for cycles 1,5 , and 10 . The average particle size varied from $732 \pm 10.4$ to $452 \pm 5.8 \mathrm{~nm}$ when the homogenization cycle was changed from 1 to 20 . The PDI also shows a gradual decrease by increasing the homogenization cycle. The PDI varied from $0.467 \pm 0.02$ to $0.332 \pm 0.01$ when the homogenization cycle was changed from 1 to 20 . The PDI was the lowest at 20 cycles.

When we compare the effect of HPMC and SDS as stabilizing agents on the average particle size, it can be seen that HPMC produces lower particle size. A similar effect was observed for the PDI also. It is known that HPMC lowers the particle size compared to other polymers such as chitosan [18]. Surfactants are more efficient as a stabilizer in producing nanosuspensions with lower particle sizes $[19,20]$.
However, in the present study, HPMC produced lower particle size than SDS. The concentration of HPMC used might be more efficient than the concentration of SDS in reducing the particle size. A similar result of better particle size and PDI for HPMC in comparison with SDS was reported in another study with celecoxib [16].

In addition to the average particle size and PDI, the zeta potential was also determined for the optimized nanosuspensions prepared at 15 cycles. HPMC-stabilized nanosuspension was having a zeta potential value of $-8.82 \pm 0.53 \mathrm{mV}$ (Figure 1(c)). HPMC-based nanosuspensions showed steric stabilization by getting adsorbed into the surface of the dispersed nanoparticles providing a steric hindrance [21]. The obtained zeta potential was reasonably good for a stable nanosuspension, and this matched with some reported values for HPMC-stabilized nanoparticles [22-24]. For the optimized SDS-stabilized 
nanosuspension, the value of the zeta potential was $-40.75 \pm 2.76 \mathrm{mV}$ (Figure 1(d)). SDS is an anionic surfactant which showed excellent dispersion properties and can produce electrostatic repulsion, and its molecules could diffuse easily into the particle surfaces resulting in excellent stabilizing effect in comparison with the HPMC-stabilized nanosuspension $[21,25]$.

\subsection{Characterization of the NAR Nanosuspension. The SEM} image showed the morphology of NAR, NAR-NS-HPMC, and NAR-NS-SDS nanosuspensions (Figures 2(a)-2(c)). Pure NAR showed a flake-like structure, heterogeneous shape, and larger particles (Figure 2(a)). NAR-NS-HPMC showed small particles, uniform particle size with smooth surface in addition to the large drug particles possibly due to the presence of dried HPMC molecules (Figure 2(b)). The SEM image of the NAR-NS-SDS nanosuspension (Figure 2(c)) did not show any clear image of small particles. Instead, some particles are seen closely placed to each other with flaky shape and larger particles. Overall, the image was similar to that of an aggregated system. Figures 2(d) and 2(e) show the TEM image of the HPMC-stabilized and SDSstabilized nanosuspensions, respectively. The TEM images were very well in accordance with the particle size analysis results. Aggregations and larger particles can be seen in the TEM image of NAR-NS-SDS, whereas the drug particles in the NAR-NS-HPMC nanosuspension appeared without any significant aggregation. The aggregation observed for NARNS-SDS may be the reason for observing a higher average particle size. The particles of the NAR-NS-HPMC nanosuspension appeared around $300 \mathrm{~nm}$ and were supporting particle size analysis results. The SEM images of the nanosuspensions were slightly different from those expected from TEM images.

3.4. Physical Stability of Nanosuspensions. The physical stability of nanosuspensions prepared using HPMC and SDS as stabilizers was assessed after storage at 4,25 , and $40^{\circ} \mathrm{C}$ for 7,14 , and 30 days. The stability data of nanosuspensions prepared using HPMC are shown in Figure 3. It can be seen that nanosuspensions prepared with HPMC have reasonable physical stabilities at $4^{\circ} \mathrm{C}$ and $25^{\circ} \mathrm{C}$. The particle size values after storage at $4^{\circ} \mathrm{C}$ were very close to each other without significant difference $(p<0.05)$. Similar observation was obtained after storage at $25^{\circ} \mathrm{C}$ also. The HPMC-stabilized nanosuspensions stored at $40^{\circ} \mathrm{C}$ exhibited slight but an observable increase in the particle size after storage for 14 and 30 days. The average particle size changed from $365 \pm 6.3$ to $399 \pm 4.4 \mathrm{~nm}$ on storage at 7 to 14 days. The probable reason for this change in particle size is the reduction in viscosity of the medium at higher temperatures leading to aggregation of suspended particles. In the case of PDI, no significant variation was observed after storage at $4^{\circ} \mathrm{C}$. Nevertheless, a slight increase in the PDI was observed after storage at $25^{\circ} \mathrm{C}$ and $40^{\circ} \mathrm{C}$. The change in the PDI was more pronounced at $4^{\circ} \mathrm{C}$ and that too after storage for 30 days. The change in the PDI may be due to the aggregation of particles to some extent. Despite a slight change in the PDI, it can be seen that the changes are not much significant to cause a drastic change in the physical stability of the system.

The stability data of nanosuspensions prepared using SDS are shown in Figure 4 . On storage at $4^{\circ} \mathrm{C}$, the average particle size was increased after storage for 14 days. However, after 30 days, it was less, but there was significant difference observed $(p<0.05)$. The results at $25^{\circ} \mathrm{C}$ were more or less similar to those observed at $4^{\circ} \mathrm{C}$, except that the particle size after 30 days was higher than that after 7 days. The observations at $4^{\circ} \mathrm{C}$ and $25^{\circ} \mathrm{C}$ could be explained based on the low solubility of SDS. The stabilizing action of SDS depends on the surface adsorption of SDS molecules on the drug nanoparticles. The aqueous solubility of SDS is less at $4^{\circ} \mathrm{C}$ and $25^{\circ} \mathrm{C}$. It is well known that SDS gets precipitated on storage at lower temperatures. Thus, on storage at low temperatures, the behaviour of SDS as a surfactant may be erratic. These might have contributed to the observations. SDS is also reported to degrade on prolonged exposure to temperature of $40^{\circ} \mathrm{C}$ or above. The observation of the particle size is in accordance with this effect. The particle size was found to increase after storage at $40^{\circ} \mathrm{C}$. After 14 days, the particle size was increased significantly. This might be due to the degradation of SDS on prolonged exposure at $40^{\circ} \mathrm{C}$, thus causing particle aggregation and an increase in the average particle size. At the same time, the PDI was not much affected on storage at $25^{\circ} \mathrm{C}$ and $40^{\circ} \mathrm{C}$ for 7,14 , and 30 days. However, there was a significant difference $(p<0.05)$ in the PDI which was observed at $4^{\circ} \mathrm{C}$. The variation in the PDI was very less compared with the average particle size. This might be due to the fact that the instability is uniformly affecting the particles thereby maintaining a similar particle size distribution. On a close examination, we can see that, on storage at $4^{\circ} \mathrm{C}$, the PDI was more erratic and can be explained by poor solubility of the surfactant to act as a stabilizing agent. Now, on a detailed analysis of the physical stability data among the two stabilizing agents, we can see that HPMC was far better than SDS at the studied concentrations. The effect of temperature was less intense in the case of HPMC-stabilized nanosuspensions stored at $4^{\circ} \mathrm{C}$ and $25^{\circ} \mathrm{C}$. In the case of nanosuspensions stored at $40^{\circ} \mathrm{C}$, both HPMC and SDS were not efficient in maintaining the stability effectively.

3.5. Solubility Studies. The equilibrium solubility of pure NAR was compared with NAR in the form of nanosuspensions stabilized using HPMC and SDS. The solubility studies were performed both in water and $\mathrm{pH} 6.8$ buffer. As expected, the results show enhancement of solubility of NAR when presented as nanosuspensions (Table 2). Similar results are reported for nanosuspensions of NAR and other poorly soluble drugs $[14,20,26]$. The extent of solubilization was comparable for both HPMC and SDS as stabilizers. Nevertheless, the solubilization of NAR was slightly more for the SDS-stabilized nanosuspension. This slight edge of SDS over HPMC could be due to the inherent solubilizing power of the former as it is a surfactant. The enhancement of solubility was noted both in water and $\mathrm{pH} 6.8$ buffer. In water, HPMC- and SDS-stabilized nanosuspensions 


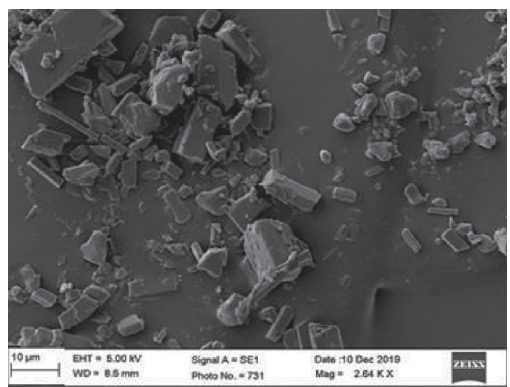

(a)

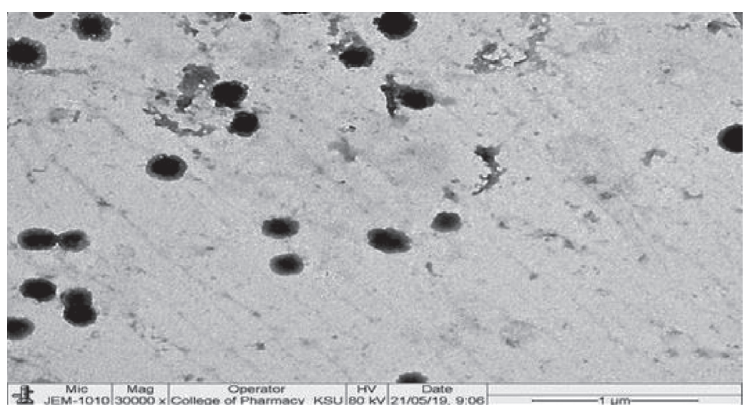

(d)

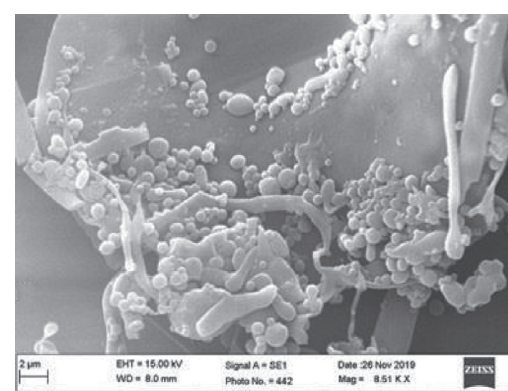

(b)

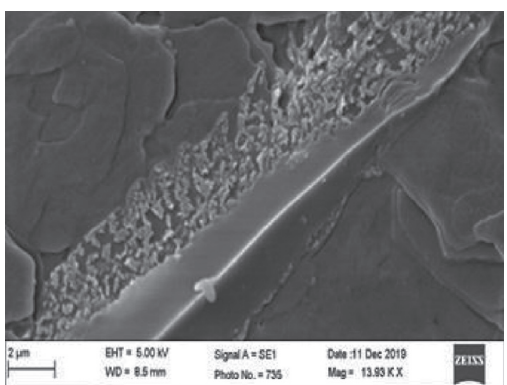

(c)

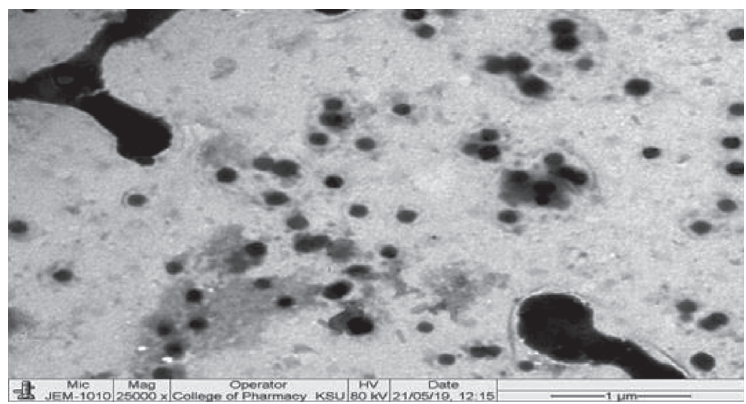

(e)

FIGURE 2: SEM micrographs of (a) pure NAR, (b) HPMC-stabilized nanosuspension, and (c) SDS-stabilized nanosuspension. (d, e) TEM images of HPMC- and SDS-stabilized nanosuspensions.

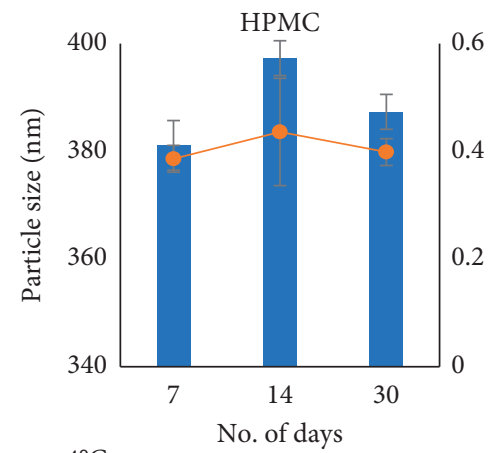

$4^{\circ} \mathrm{C}$

$$
\rightarrow \text { PDI }
$$

HPMC

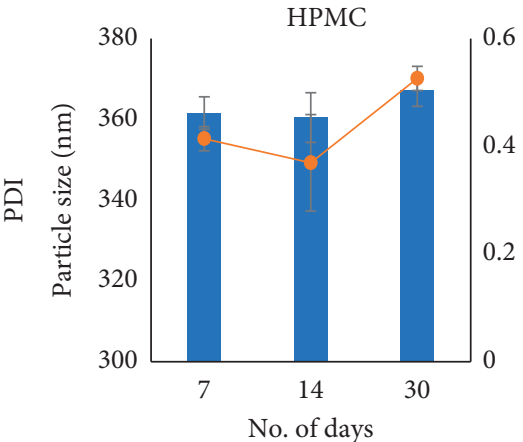

$25^{\circ} \mathrm{C}$

$$
\rightarrow \text { PDI }
$$

HPMC

$$
\text { ลี }
$$

$40^{\circ} \mathrm{C}$

$$
\multimap \text { PDI }
$$

FIGURE 3: Stability profile of the HPMC-stabilized naringenin nanosuspension as a function of number of days at different storage temperatures (after 15 homogenization cycles, pressure 1500 bar and $n=3$ ).

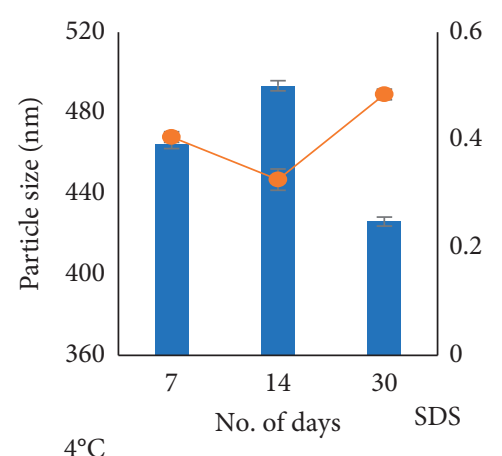

$4^{\circ} \mathrm{C}$

$$
\rightarrow \text { PDI }
$$

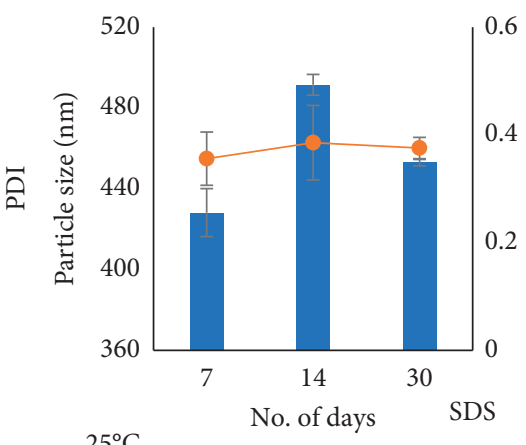

$25^{\circ} \mathrm{C}$

$$
\rightarrow \text { PDI }
$$

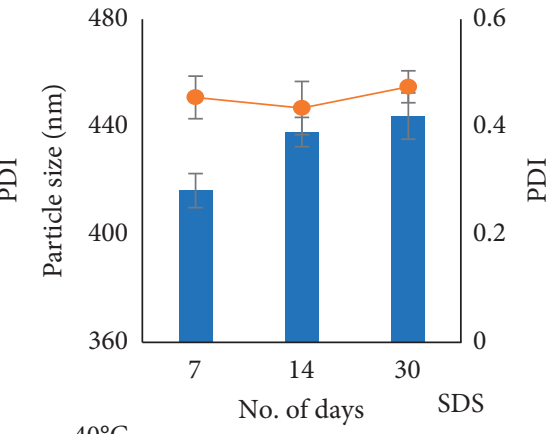

$40^{\circ} \mathrm{C}$

$$
\rightarrow \text { PDI }
$$

FIGURE 4: Stability profile of the SDS-stabilized naringenin nanosuspension as a function of number of days at different storage temperatures (after 15 homogenization cycles, pressure 1500 bar and $n=3$ ). 
TABLE 2: The saturation solubility of naringenin solution (NAR) and naringenin nanosuspensions in distilled water and phosphate buffer ( $\mathrm{pH}$ 6.8).

\begin{tabular}{lcc}
\hline Formulations & $\begin{array}{c}\text { Distilled water } \\
(\mu \mathrm{g} / \mathrm{mL})\end{array}$ & $\begin{array}{c}\text { Phosphate buffer } \\
(\mathrm{pH} \mathrm{6.8)}(\mu \mathrm{g} / \mathrm{mL})\end{array}$ \\
\hline NAR solution & $34 \pm 6.2$ & $18 \pm 2.6$ \\
NAR-NS-HPMC & $925 \pm 9.5$ & $713 \pm 8.4$ \\
NAR-NS-SDS & $977 \pm 13.3$ & $765 \pm 13.8$ \\
\hline
\end{tabular}

produced enhancement of solubility of around 27.2 and 28.7 times, respectively. The enhancement of solubility by HPMC and SDS was more in $\mathrm{pH} 6.8$ buffer and was around 39.7 and 42.6 times, respectively. The solubility of pure NAR was less in $\mathrm{pH} 6.8$ buffer compared with water.

3.6. In Vitro Release Study. The in vitro release study was performed in PBS (pH 7.4) buffer with pure NAR, HPMCstabilized nanosuspension, and SDS-stabilized nanosuspension by the dialysis bag method (Figure 5). The results of the release study were slightly different than expected from the saturation solubility results. Some reported nanosuspensions show an initial burst release [25]. However, a typical burst release was neither observed from HPMC-stabilized nor SDS-stabilized nanosuspensions. A gradual drug release was observed from both HPMC- and SDS-stabilized nanosuspensions. The drug release from HPMC- and SDS-stabilized nanosuspensions was more or less similar up to $60 \mathrm{~min}$. Later, the drug release was more from the HPMC-stabilized nanosuspension and reached $89.2 \pm 5.4 \%$ at $120 \mathrm{~min}$. At the same time, the SDS-stabilized nanosuspension could release only $71.3 \pm 3.27 \%$ of the drug. A lower release from the SDS-stabilized nanosuspension might be due to the escape of SDS molecules into the release medium thereby causing a reduction in the number of molecules to solubilize NAR. In the case of HPMC, this was not possible due to high molecular weight than the cut-off value of the dialysis bag. The drug release from the pure NAR powder was very low compared with that of the nanosuspensions. This result was expected and was observed in many studies $[16,20]$.

3.7. Determination of Antioxidant Activity. The antioxidant effect of NAR, NAR-NS-SDS, and NAR-NS-HPMC was determined in comparison with gallic acid (Figure 6). Gallic acid is very common as a standard in the DPPH assay $[27,28]$. The antioxidant effect was measured in terms of inhibition of the DPPH free radical. On increasing the concentration of DPPH, the antioxidant effect of the samples was found to decrease. In all the cases, the antioxidant effect of the SDS-stabilized nanosuspension was slightly high in the mean value than the HPMC-stabilized nanosuspension. However, the difference was not significant. The difference in antioxidant activity was more visible at DPPH concentrations of 10 and $20 \mu \mathrm{g} / \mathrm{mL}$. A significant difference was obtained for stabilized nanosuspensions and pure NAR. At a

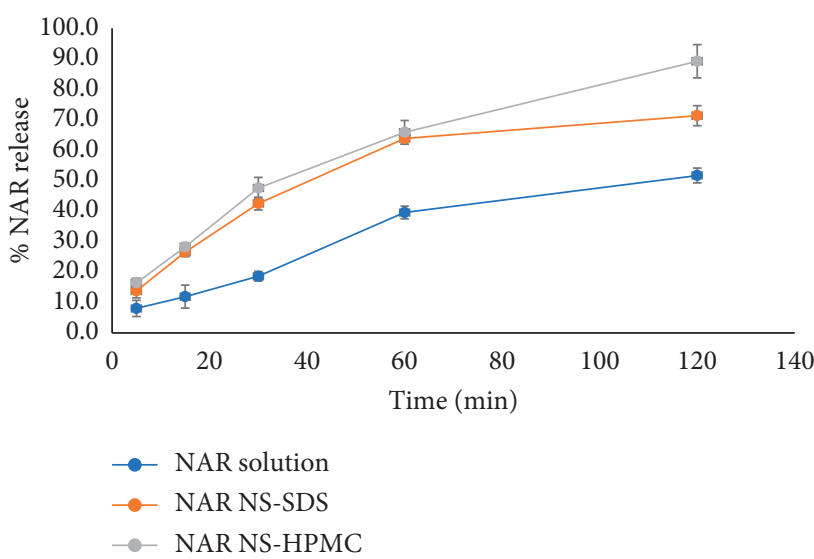

FIgURE 5: Comparative dissolution profile of naringenin and naringenin nanosuspensions in phosphate-buffered solution $(\mathrm{pH}$ $6.8)$ at $37 \pm 0.5^{\circ} \mathrm{C}(n=3)$.

DPPH concentration of 100, the (\%) inhibition was similar for NAR, NAR-NS-SDS, and NAR-NS-HPMC.

3.8. In Vitro Cytotoxicity Assessment. Cytotoxicity of the samples was studied in MCF-7 breast cancer cells by using the MTT assay. NAR suspension and nanosuspensions (NAR-NS-SDS and NAR-NS-HPMC) were studied to monitor their cytotoxicity on cell lines. The study used NAR concentrations equivalent to $10,20,30,50$, and $100 \mu \mathrm{g} / \mathrm{mL}$. The cytotoxicity assessment of HPMC and SDS was studied as a negative control to rule out the effect of excipients on the cytotoxicity result. It was shown that, after 24 and $48 \mathrm{~h}$ of incubation with $100 \mu \mathrm{g} / \mathrm{mL}$ of HPMC and SDS, the cell viability was more than $95 \%$. These results indicated that HPMC and SDS used in the experiment had no effect on the cytotoxicity and can be used as safe stabilizers. The results show promising cytotoxic effect on cancer cells and indicated antitumor potential for the NAR nanosuspensions (Figure 7). After $24 \mathrm{~h}$, at all studied concentrations, the lowest cytotoxicity was observed for NAR solution. At lower drug concentrations, the difference in cytotoxicity among the NAR solution and nanosuspensions was not prominent. However, on increasing the drug concentration, the effect became prominent. Drug concentration higher than $30 \mu \mathrm{g} / \mathrm{mL}$ produced significant $(p<0.05)$ cytotoxicity after $24 \mathrm{~h}$ when presented as NAR nanosuspensions. The incubation time also increased the cytotoxicity of all the samples. Thus, the (\%) cell viability values for all the samples after $48 \mathrm{~h}$ were less than those at $24 \mathrm{~h}$. An interesting observation in the cytotoxicity studies was the effect of nanosuspensions. It is observed that, at higher drug concentration and increased incubation time, the HPMCstabilized nanosuspensions (NAR-NS-HPMC) were more cytotoxic than SDS-stabilized nanosuspensions (NAR-NSSDS). At the highest studied drug concentration of $100 \mu \mathrm{g} / \mathrm{mL}$ and the maximum studied incubation time of $48 \mathrm{~h}$, both nanosuspensions produced similar cytotoxicity. IC50 for NAR and NAR nanosuspensions was reduced with increased time of treatment (Figure 7). IC50 of the NAR nanosuspension was much lower than that of the NAR solution after the same 


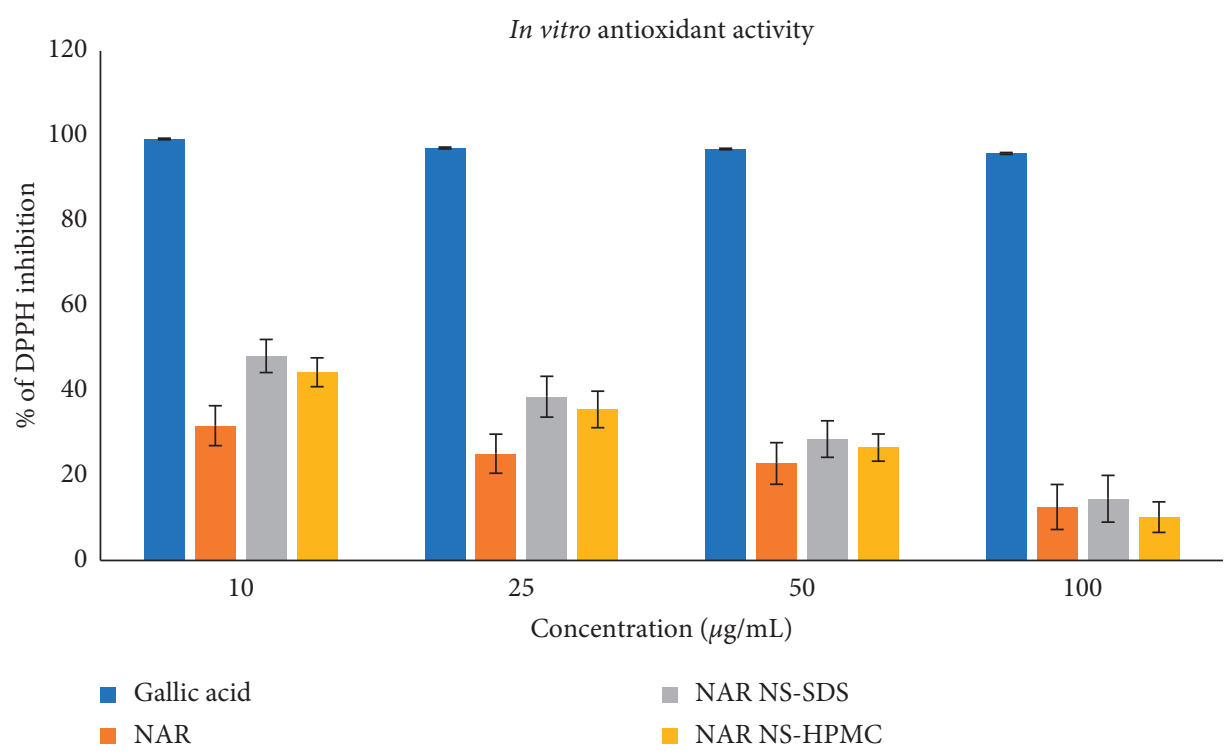

FIGURE 6: Percentage of DPPH free radical inhibition of NAR, SDS-stabilized NAR nanosuspension (NAR-NS-SDS), and HPMC-stabilized NAR nanosuspension (NAR-NS-HPMC) compared with standard gallic acid as a function of different concentrations of DPPH.
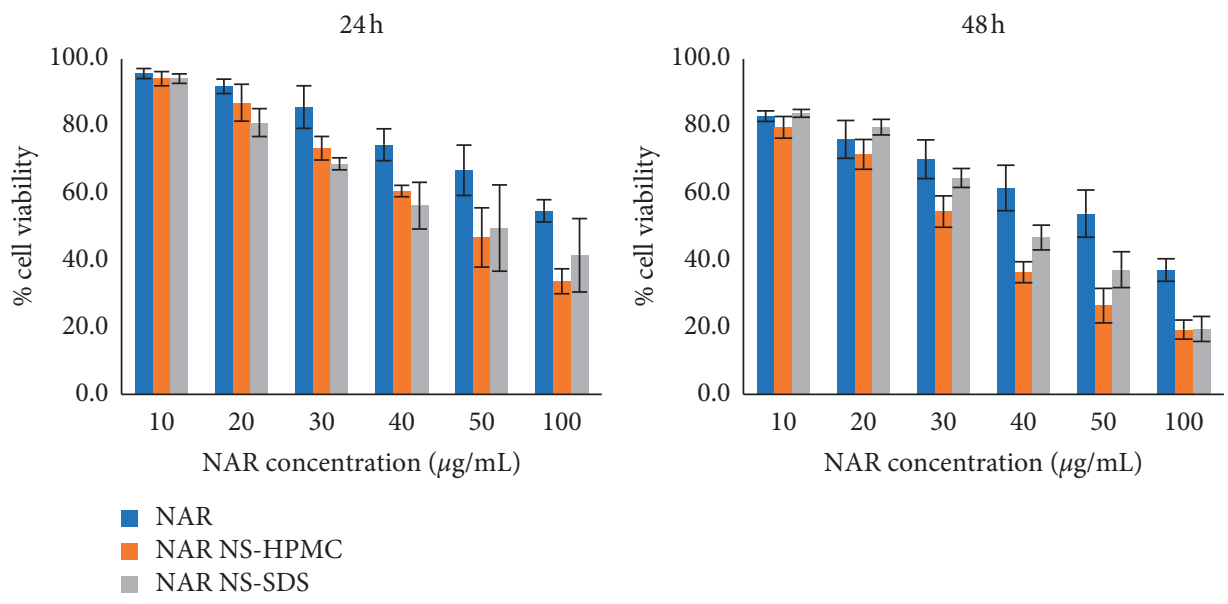

\begin{tabular}{|c|c|c|}
\hline Formulations & $24 \mathrm{~h}$ & $48 \mathrm{~h}$ \\
\hline NAR solution & 103.7 & 80.81 \\
\hline NAR NS-HPMC & 66.58 & 48.01 \\
\hline NAR NS-SDS & 71.18 & 57.25 \\
\hline
\end{tabular}

FIGURE 7: MTT assay showing effects of free naringenin and HPMC- and SDS-stabilized naringenin nanosuspensions on the cell viability of MCF-7 cells in a time- and dose-dependent manner. Data are expressed as the mean \pm SD of three independent experiments $(n=3)$.

incubation time (Figure 7). These results indicated that the NAR nanosuspension had an enhanced efficacy as compared with NAR solution. The improved cytotoxicity of the NAR nanosuspension compared with NAR solution is due to increased solubility and dissolution rates of NAR nanosuspension resulting in higher molecular concentration around the cells. This nanosuspension can enter cells via endocytosis or phagocytosis resulting in higher accumulation within the cells. These factors lead to higher efficacy of NAR nanosuspension $[29,30]$. Similar enhancement of cytotoxicity by the formulation of nanosuspension has been reported for naringenin and other drugs $[29,30]$.
3.9. Morphological Analysis of Cells by Phase-Contrast Microscopy. The morphology of the incubated cells was in accordance with the cytotoxicity results by using the MTT assay. All the samples show time-dependent morphological changes on observation with a phase-contrast microscope (Figure 8). Cell shrinkage and formation of apoptotic bodies were noted on incubation with NAR, NAR-NS-SDS, and NAR-NS-HPMC. Cell shrinkage and formation of apoptotic bodies were more prominent after $48 \mathrm{~h}$ of incubation. From the images, these effects were more pronounced for the HPMC-stabilized nanosuspension. These types of morphological changes were observed for MCF-7 breast cancer 


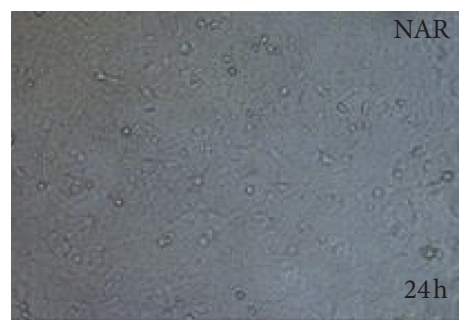

(a)

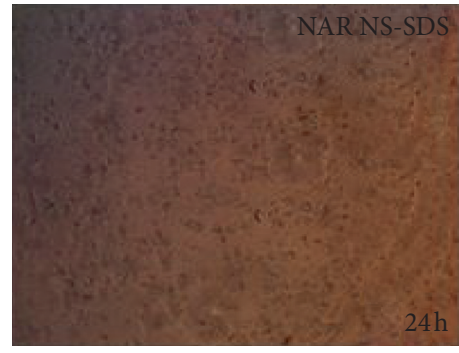

(d)

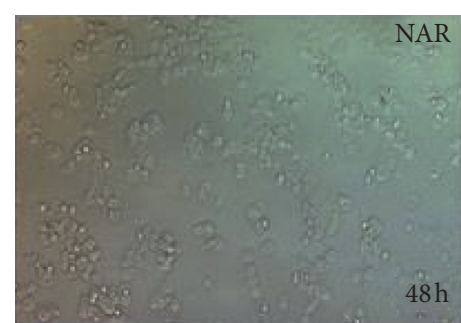

(b)

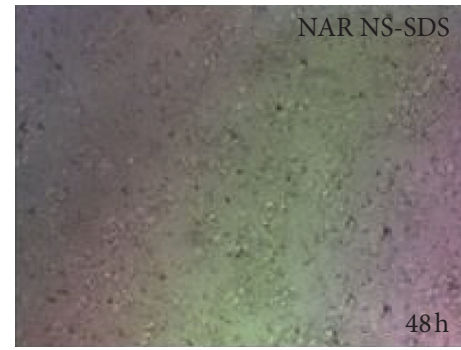

(e)

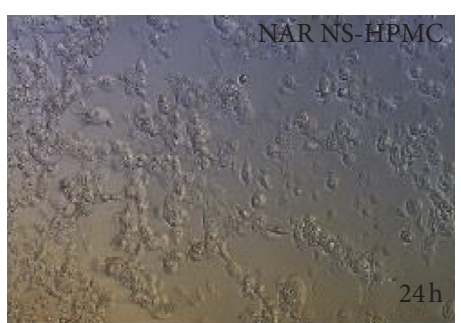

(c)

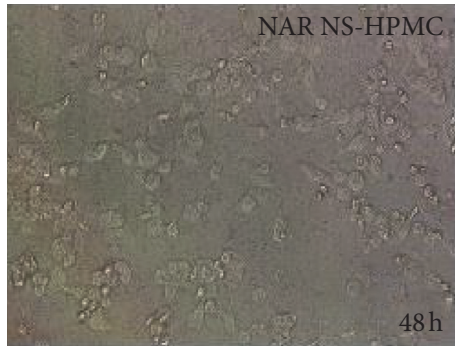

(f)

FIGURE 8: MCF-7 cells were incubated with naringenin solution, HPMC-, and SDS-stabilized naringenin nanosuspension formulations at $100 \mu \mathrm{g} / \mathrm{ml}$ to induce morphological changes: (a) 24 hours (NAR); (b) 48 hours (NAR); (c) 24 hours (NAR-NS-HPMC); (d) 24 hours (NARNS-SDS); (e) 48 hours (NAR-NS-SDS); (f) 48 hours (NAR-NS-HPMC).

cells with other cytotoxic nanostructures and extracts [31-33].

\section{Conclusion}

HPMC- and SDS-stabilized nanosuspensions were prepared by premilling and subsequent high-pressure homogenization of NAR. HPMC and SDS were evaluated for their performance as stabilizers under homogenization cycles of $1,5,10,15$, and 20 . The prepared nanosuspensions were studied for particle size and size distribution, zeta potential, solubility, drug release, antioxidant activity, and in vitro antitumor activity. The particle size and size distribution were determined by PCS. The homogenization cycle was found to affect the average particle size and the PDI. Lower particle size was obtained for NAR-NS-HPMC when compared with NAR-NS-SDS. Physical stability of NAR-NS-HPMC was better when compared with that of NAR-NS-SDS. Temperature-dependent instability was observed for NAR-NS-SDS. Meanwhile, the solubility enhancement of the drug from nanosuspensions of both stabilizers was comparable. The antioxidant effect of both nanosuspensions was comparable. However, a slightly higher mean value was noted for NAR-NS-SDS. Both NARNS-HPMC and NAR-NS-SDS produced enhanced in vitro cytotoxicity compared with NAR. Overall, NAR-NSHPMC was found to be better than NAR-NS-SDS in terms of particle size and size distribution, zeta potential, storage stability, and drug release.

\section{Data Availability}

The data used to support the findings of this study are included within the article.

\section{Conflicts of Interest}

The authors declare that they have no conflicts of interest.

\section{Acknowledgments}

This project was funded by the Deanship of Scientific Research (DSR) at King Abdulaziz University, Jeddah under grant no. RG: 5-166-40. The authors, therefore, acknowledge with thanks DSR for technical and financial support.

\section{References}

[1] M. Carvalho, C. Jerónimo, P. Valentão, P. B. Andrade, and B. M. Silva, "Green tea: a promising anticancer agent for renal cell carcinoma," Food Chemistry, vol. 122, no. 1, pp. 49-54, 2010.

[2] M. Abaza, K. Orabi, E. Al-Quattan, and R. Al-Attiyah, "Growth inhibitory and chemo-sensitization effects of naringenin, a natural flavanone purified from Thymus vulgaris, on human breast and colorectal cancer," Cancer Cell International, vol. 15, no. 1, 2015.

[3] A. C. Tan, I. Konczak, D. M. Sze, and I. Ramzan, "Molecular pathways for cancer chemoprevention by dietary phytochemicals," Nutrition and Cancer, vol. 63, pp. 495-505, 2012.

[4] K. W. Wahle, I. Brown, D. Rotondo, and S. D. Heys, "Plant phenolics in the prevention and treatment of cancer," Advances in Experimental Medicine and Biology, vol. 698, pp. 36-51, 2011.

[5] N. Krishnakumar, N. Sulfikkarali, N. RajendraPrasad, and S. Karthikeyan, "Enhanced anticancer activity of naringeninloaded nanoparticles in human cervical (HeLa) cancer cells," Biomedicine \& Preventive Nutrition, vol. 1, no. 4, pp. 223-231, 2011.

[6] F.-L. Yen, T.-H. Wu, L.-T. Lin, T.-M. Cham, and C.-C. Lin, "Naringenin-loaded nanoparticles improve the physicochemical properties and the hepatoprotective effects of 
naringenin in orally-administered rats with CCl4-induced acute liver failure," Pharmaceutical Research, vol. 26, no. 4, pp. 893-902, 2009.

[7] V. R. Patel and Y. K. Agrawal, "Nanosuspension: an approach to enhance solubility of drugs," Journal of Advanced Pharmaceutical Technology \&amp; Research, vol. 2, no. 2, pp. 8187, 2011.

[8] L. Al Shaal, R. Shegokar, and R. H. Müller, "Production and characterization of antioxidant apigenin nanocrystals as a novel UV skin protective formulation," International Journal of Pharmaceutics, vol. 420, no. 1, pp. 133-140, 2011.

[9] P. Kocbek, S. Baumgartner, and J. Kristl, "Preparation and evaluation of nanosuspensions for enhancing the dissolution of poorly soluble drugs," International Journal of Pharmaceutics, vol. 312, no. 1-2, pp. 179-186, 2006.

[10] Y. Wang, Y. Ma, Y. Zheng et al., "In vitro and in vivo anticancer activity of a novel puerarin nanosuspension against colon cancer, with high efficacy and low toxicity," International Journal of Pharmaceutics, vol. 441, no. 1-2, pp. 728-735, 2013.

[11] M. S. Freag, Y. S. R. Elnaggar, and O. Y. Abdallah, "Development of novel polymer-stabilized diosmin nanosuspensions: in vitro appraisal and ex vivo permeation," International Journal of Pharmaceutics, vol. 454, no. 1, pp. 462-471, 2013.

[12] P. Venkatesan, N. Puvvada, R. Dash et al., "The potential of celecoxib-loaded hydroxyapatite-chitosan nanocomposite for the treatment of colon cancer," Biomaterials, vol. 32 , no. 15 , pp. 3794-3806, 2011.

[13] P. R. Mishra, L. A. Shaal, R. H. Müller, and C. M. Keck, "Production and characterization of Hesperetin nanosuspensions for dermal delivery," International Journal of Pharmaceutics, vol. 371, no. 1-2, pp. 182-189, 2009.

[14] E. Ahire, S. Thakkar, M. Darshanwad, and M. Misra, "Parenteral nanosuspensions: a brief review from solubility enhancement to more novel and specific applications," Acta Pharmaceutica Sinica B, vol. 8, no. 5, pp. 733-755, 2018.

[15] A. M. Cerdeira, M. Mazzotti, and B. Gander, "Formulation and drying of miconazole and itraconazole nanosuspensions," International Journal of Pharmaceutics, vol. 443, no. 1-2, pp. 209-220, 2013.

[16] J. He, Y. Han, G. Xu et al., "Preparation and evaluation of celecoxib nanosuspensions for bioavailability enhancement," RSC Advances, vol. 7, no. 22, pp. 13053-13064, 2017.

[17] J. Ma, Y. Yang, Y. Sun, and J. Sun, "Optimization, characterization and in vitro/vivo evaluation of azilsartan nanocrystals," Asian Journal of Pharmaceutical Sciences, vol. 12, pp. 344-352, 2016.

[18] N. P. Ambhore, P. M. Dandagi, and A. P. Gadad, "Formulation and comparative evaluation of HPMC and water soluble chitosan-based sparfloxacin nanosuspension for ophthalmic delivery," Drug Delivery and Translational Research, vol. 6, no. 1, pp. 48-56, 2016.

[19] F. Li, L. Li, S. Wang et al., "Improved dissolution and oral absorption by co-grinding active drug probucol and ternary stabilizers mixtures with planetary beads-milling method," Asian Journal of Pharmaceutical Sciences, 2018.

[20] M. K. Singh, D. Pooja, H. G. Ravuri, A. Gunukula, H. Kulhari, and R. Sistla, "Fabrication of surfactant-stabilized nanosuspension of naringenin to surpass its poor physiochemical properties and low oral bioavailability," Phytomedicine, vol. 40, pp. 48-54, 2018.

[21] M. A. Ibrahim, G. A. Shazly, F. S. Aleanizy, F. Y. Alqahtani, and G. M. Elosaily, "Formulation and evaluation of docetaxel nanosuspensions: in-vitro evaluation and cytotoxicity," Saudi Pharmaceutical Journal, vol. 27, no. 1, pp. 49-55, 2019.

[22] S. Md, S. Jagdish, D. David, M. Pandey, and L. Chatterjee, "Development and in vitro evaluation of a zerumbone loaded nanosuspension drug delivery system," Crystals, vol. 8, no. 7, p. 286, 2018.

[23] A. A. Abdelbary, X. Li, M. El-Nabarawi, A. Elassasy, and B. Jasti, "Effect of fixed aqueous layer thickness of polymeric stabilizers on zeta potential and stability of aripiprazole nanosuspensions," Pharmaceutical Development and Technology, vol. 18, no. 3, pp. 730-735, 2013.

[24] D. H. Alshora, M. A. Ibrahim, E. Elzayat, O. T. Almeanazel, and F. Alanazi, "Rosuvastatin calcium nanoparticles: improving bioavailability by formulation and stabilization codesign," PLoS One, vol. 13, Article ID e0200218, 2018.

[25] T. Geng, P. Banerjee, Z. Lu, A. Zoghbi, T. Li, and B. Wang, "Comparative study on stabilizing ability of food protein, non-ionic surfactant and anionic surfactant on BCS type II drug carvedilol loaded nanosuspension: physicochemical and pharmacokinetic investigation," European Journal of Pharmaceutical Sciences, vol. 109, pp. 200-208, 2017.

[26] V. R. Patel and Y. K. Agrawal, "Nanosuspension: an approach to enhance solubility of drugs," Journal of Advanced Pharmaceutical Technology \&amp; Research, vol. 2, no. 2, pp. 8187, 2011, https://doi.org/10.4103/2231-4040.82950.

[27] S. Kumar, R. Sandhir, and S. Ojha, "Evaluation of antioxidant activity and total phenol in different varieties of Lantana camara leaves," BMC Research Notes, vol. 7, no. 1, p. 560, 2014.

[28] J. O. Olugbami, M. A. Gbadegesin, and O. A. Odunola, "In vitro free radical scavenging and antioxidant properties of ethanol extract of Terminalia glaucescens," Pharmacognosy Res, vol. 7, pp. 49-56, 2015.

[29] F.-F. Feng, D.-R. Zhang, K.-L. Tian et al., "Growth inhibition and induction of apoptosis in MCF-7 breast cancer cells by oridonin nanosuspension," Drug Delivery, vol. 18, no. 4, pp. 265-271, 2011.

[30] S. Rajamani, A. Radhakrishnan, T. Sengodan, and S. Thangavelu, "Augmented anticancer activity of naringeninloaded TPGS polymeric nanosuspension for drug resistive MCF-7 human breast cancer cells," Drug Development and Industrial Pharmacy, vol. 44, no. 11, pp. 1752-1761, 2018.

[31] R. Abbasalipourkabir, A. Salehzadeh, and R. Abdullah, “Tamoxifen-loaded solid lipid nanoparticles-induced apoptosis in breast cancer cell lines," Journal of Experimental Nanoscience, vol. 11, no. 3, pp. 161-174, 2016.

[32] J. Murugaraj, A. Renganathan, S. Gnanasekar, K. Premkumar, and A. Ganapathi, "Biogenic metal nanoformulations induce $\mathrm{Bax} / \mathrm{Bcl} 2$ and caspase mediated mitochondrial dysfunction in human breast cancer cells (MCF 7)," RSC Advance, vol. 5, 2014.

[33] D. Silva, F. Vidal, D. Santos et al., "Cytotoxic effects of Euterpe oleracea Mart. in malignant cell lines," BMC Complement. Altern. Med.vol. 14, p. 175, 2014. 